\title{
Strong CP violation in spin-1/2 singly charmed baryons
}

\author{
Y. Ünal ${ }^{a, b}$ and UIf-G. Meißner ${ }^{a, c, d}$ \\ ${ }^{a}$ Helmholtz-Institut für Strahlen- und Kernphysik and \\ Bethe Center for Theoretical Physics Universität Bonn, \\ D-53115 Bonn, Germany \\ ${ }^{b}$ Physics Department, Çanakkale Onsekiz Mart University, \\ 17100 Çanakkale, Turkey \\ ${ }^{c}$ Institute for Advanced Simulation, Institut für Kernphysik and \\ Jülich Center for Hadron Physics, \\ Forschungszentrum Jülich, D-52425 Jülich, Germany \\ ${ }^{d}$ Tbilisi State University, \\ 0186 Tbilisi, Georgia \\ E-mail: unal@hiskp.uni-bonn.de, meissner@hiskp.uni-bonn.de
}

ABSTRACT: We report on the calculation of the CP-violating form factor $F_{3}$ and the corresponding electric dipole moment for charmed baryons in the spin- $1 / 2$ sector generated by the QCD $\theta$-term. We work in the framework of covariant baryon chiral perturbation theory within the extended-on-mass-shell renormalization scheme up to next-to-leading order in the chiral expansion.

KEYwords: CP violation, Chiral Lagrangians, Effective Field Theories

ArXiv EPrint: 2008.01371 


\section{Contents}

1 Introduction 1

2 Chiral Lagrangian including CP-violating terms 2

3 CP-violating electromagnetic form factor 5

4 Results $\quad 9$

5 Conclusion $\quad 9$

$\begin{array}{ll}\text { A Scalar loop integrals } & 10\end{array}$

B Loop contributions 10

C Results for the CP-violating form factor $\quad 14$

\section{Introduction}

Charge conjugation and parity symmetry $(\mathrm{CP})$ violation is an essential condition for an asymmetry between matter and anti-matter in the present universe. On the other hand, CP violation by the complex phase of the Cabibbo-Kobayashi-Maskawa (CKM) quark-mixing matrix is insufficient to explain the dominance of matter over antimatter [1,2], meaning that the presence of other $\mathrm{CP}$ violating mechanisms within or beyond the Standard Model (SM) is required. The QCD $\theta$-term is the only source of $\mathrm{P}$ and $\mathrm{T}$ violation within the SM beyond the complex phase of the CKM quark-mixing matrix. However, because of the significant suppression of electric dipole moments (EDMs) induced by the complex phase of the CKM matrix and unobservably small CKM backgrounds, any measurement of EDM of any quantum system would indicate of presence $\mathrm{CP}$ violation beyond the CKM mechanism in the SM. EDMs are important observables generated by the CP-violating effects. Thus, measurements of hadron EDMs lead to severe restrictions in the mechanism generating CP violation, as detailed e.g. in ref. [3].

$\mathrm{CP}$ violation has recently been established in the charm sector, more precisely in the meson decays $D^{0} \rightarrow K^{-} K^{+}$and $D^{0} \rightarrow \pi^{-} \pi^{+}$[4], and LHCb has also measured the difference of CP-asymmetry of the three-body singly Cabibbo-suppressed $\Lambda_{c}^{+}$decays [5]. There have also been quite a number of studies predicting $\mathrm{CP}$ asymmetries in charmed baryon decays, see e.g. [6] and references therein. It is therefore of interest to investigate other possible effects of $\mathrm{CP}$ violation in singly-charmed baryons. Indeed, a first measurement of $\mathrm{CP}$ violation in $\Xi_{c}^{+} \rightarrow p K^{-} \pi^{+}$decays has been performed by LHCb [7]. However, these data are consistent with the hypothesis of no $\mathrm{CP}$ violation. On the other hand, another experimental study promises to search for direct $\mathrm{CP}$ violation by measuring the asymmetries of three different decay channels of the $\Lambda_{c}^{+}$baryon [8].

Here, we concentrate on the effects generated by the strong CP-violating $\theta$-term of $\mathrm{QCD}$, that also induces electric dipole moments in light baryons, as pioneered in 
refs. [10, 11]. The proper framework to address such questions is baryon chiral perturbation theory, see [12] for a review. In fact, the masses, axial charges, and electromagnetic decays of the charmed and bottomed baryons have already been calculated in the framework of the heavy baryon approach $[13,14]$. More recently, the magnetic moments of the spin-1/2 singly charmed baryons were analyzed in covariant baryon chiral perturbation theory [15]. In this paper, we extend these studies and work out the CP-violating effects induced by the QCD $\theta$-term. While there is experimental activity to assess the effects of the $\theta$-term in neutron and proton EDMs, singly-charmed baryons offer a completely new venue towards these elusive effects, with very different systematic uncertainties that hamper such measurements. How competitive these measurements will be would require a much more refined analysis as presented here. We note that recent progress towards the first measurement of the charm baryon dipole moments has been reported in ref. [9], thus our investigation is very timely. In the near future, further measurements with charmed hadrons, along with different theoretical improvements, would help to further elucidate the $\mathrm{CP}$ violation in the charm quark sector.

The manuscript is organized as follows. In section 2, we briefly discuss the underlying chiral Lagrangian. The CP-violating electromagnetic form factor of the singly-charmed baryons is worked out in section 3 followed by the display of our numerical results in section 4 . Section 5 contains the summary and outlook. The appendices contain some technicalities as well as more detailed tables of results.

\section{Chiral Lagrangian including CP-violating terms}

The QCD Lagrangian of the strong interactions including the $\theta$ term reads

$$
\mathcal{L}_{\mathrm{QCD}}=\bar{q}(i \not D-\mathcal{M}) q-\frac{1}{4} \mathcal{G}_{a \mu \nu} \mathcal{G}_{a}^{\mu \nu}+\frac{g^{2} \theta}{64 \pi^{2}} \varepsilon_{\mu \nu \rho \sigma} \mathcal{G}_{a}^{\mu \nu} \mathcal{G}_{a}^{\rho \sigma}, \quad a=1, \ldots, 8,
$$

where $\mathcal{G}_{a}^{\mu \nu}$ is the gluon field-strength tensor, $g$ is the strong coupling constant and $\mathcal{M}$ is the quark mass matrix. Strong CP violation arising from the U(1) anomaly in QCD is specified via the vacuum angle $\theta$. The measurable quantity is not $\theta$ but the combination

$$
\theta_{0}=\theta+\arg \operatorname{det} \mathcal{M},
$$

because of the anomaly. Here, to describe the phenomena related to the $\theta$-term, we seek a description in a properly tailored effective field theory, see e.g. refs. [16, 17] for the detailed construction of the corresponding effective Lagrangian to one loop accuracy.

The Goldstone bosons together with the flavor singlet $\eta_{0}$, resulting from the spontaneous symmetry breaking of $\mathrm{U}(3)_{R} \times \mathrm{U}(3)_{L}$ into $\mathrm{U}(1)_{V}$, are represented by the matrixvalued field $\tilde{U}$. Treating the vacuum angle $\theta(x)$ as an external field, it transforms as $\theta(x) \rightarrow \theta^{\prime}(x)=\theta(x)-2 N_{f} \alpha$ under axial U(1) rotations, with $N_{f}$ the number of flavors, and $\alpha$ is the rotation angle. Following the spontaneous chiral symmetry breaking, under the axial $\mathrm{U}(1)$ transformation, $\tilde{U}$ changes but the combination of $\bar{\theta}_{0}(x)=\theta(x)-i \ln \operatorname{det} \tilde{U}(x)$ stays invariant. Using this invariant combination of $\bar{\theta}_{0}(x)$, one can construct the most general mesonic chiral effective Lagrangian up-to-and-including second chiral order

$$
\begin{aligned}
\mathcal{L}= & -V_{0}+V_{1}\left\langle\nabla_{\mu} \tilde{U}^{\dagger} \nabla^{\mu} \tilde{U}\right\rangle+V_{2}\left\langle\tilde{\chi} \tilde{U}+\tilde{\chi} \tilde{U}^{\dagger}\right\rangle+i V_{3}\left\langle\tilde{\chi} \tilde{U}-\tilde{\chi} \tilde{U}^{\dagger}\right\rangle \\
& +V_{4}\left\langle\tilde{U} \nabla_{\mu} \tilde{U}^{\dagger}\right\rangle\left\langle\tilde{U}^{\dagger} \nabla^{\mu} \tilde{U}\right\rangle .
\end{aligned}
$$


Note that $\langle\ldots\rangle$ denotes the trace in flavor space and $\tilde{\chi}=2 B_{0} \mathcal{M}$, with the light quark mass matrix $\mathcal{M}=\operatorname{diag}\left(m_{u}, m_{d}, m_{s}\right)$. The covariant derivative of $\tilde{U}$ is given by

$$
\nabla_{\mu} \tilde{U}=\partial_{\mu} \tilde{U}-i\left(v_{\mu}+a_{\mu}\right) \tilde{U}+i \tilde{U}\left(v_{\mu}-a_{\mu}\right)
$$

where $v_{\mu}$ and $a_{\mu}$ are the conventional vector and axial-vector external sources. The $V_{i}$ coefficients in the Lagrangian (2.3) are functions of $\bar{\theta}_{0}$. One needs to determine the vacuum expectation value of $\tilde{U}$ in order to include non-trivial vacuum effects based on the angle $\theta_{0}$. Parameterizing the vacuum as

$$
U_{0}=\operatorname{diag}\left(e^{-i \varphi_{u}}, e^{-i \varphi_{d}}, e^{-i \varphi_{s}}\right),
$$

the minimized potential energy $V\left(U_{0}\right)$ can be determined using the notation $\bar{\theta}_{0}=\theta_{0}-$ $\sum_{q} \varphi_{q}$. In this way, the Taylor expansions of the $V_{i}$ functions in terms of $\bar{\theta}_{0}$ yield

$$
\begin{aligned}
V_{i}\left(\bar{\theta}_{0}\right) & =\sum_{n=0}^{\infty} V_{i}^{(2 n)} \bar{\theta}_{0}^{2 n} \quad \text { for } \quad i=0,1,2,4 \\
V_{3}\left(\bar{\theta}_{0}\right) & =\sum_{n=0}^{\infty} V_{i}^{(2 n+1)} \bar{\theta}_{0}^{2 n+1} .
\end{aligned}
$$

Note that while all other $V_{i}$ are even function of $\bar{\theta}_{0}, V_{3}$ is odd. To express the Lagrangian in terms of the angles $\varphi_{q}$ one then writes the $\tilde{U}$ with the vacuum expectation value $U_{0}$ as $\tilde{U}=\sqrt{U_{0}} U \sqrt{U_{0}}$ by choosing

$$
U=\exp \left(i \sqrt{\frac{2}{3}} \frac{\eta_{0}}{F_{0}}+i \frac{\sqrt{2}}{F_{\pi}} \phi\right),
$$

where $\phi$ represents the Goldstone boson octet

$$
\phi=\left(\begin{array}{ccc}
\frac{1}{\sqrt{2}} \pi^{0}+\frac{1}{\sqrt{6}} \eta_{8} & \pi^{+} & K^{+} \\
\pi^{-} & -\frac{1}{\sqrt{2}} \pi^{0}+\frac{1}{\sqrt{6}} \eta_{8} & K^{0} \\
K^{-} & \bar{K}^{0} & -\frac{2}{\sqrt{6}} \eta_{8}
\end{array}\right) .
$$

Thus, the chiral effective Lagrangian in terms of the Goldstone boson fields composed in $\tilde{U}$ reads $[18]$

$$
\begin{aligned}
\mathcal{L}_{\phi}= & -V_{0}+V_{1}\left\langle\nabla_{\mu} U^{\dagger} \nabla^{\mu} U\right\rangle+\left(V_{2}+\mathcal{B} V_{3}\right)\left\langle\chi\left(U+U^{\dagger}\right)\right\rangle-i \mathcal{A} V_{2}\left\langle U-U^{\dagger}\right\rangle \\
& +\mathcal{A} V_{3}\left\langle U+U^{\dagger}\right\rangle+V_{4}\left\langle U \nabla_{\mu} U^{\dagger}\right\rangle\left\langle U^{\dagger} \nabla^{\mu} U\right\rangle .
\end{aligned}
$$

Here $\chi=2 B_{0} \operatorname{diag}\left(m_{u} \cos \varphi_{u}, m_{d} \cos \varphi_{d}, m_{s} \cos \varphi_{s}\right)$. To leading order, $\mathcal{A}$ and $\mathcal{B}$ are given as

$$
\mathcal{A}=\frac{V_{0}^{(2)}}{V_{2}^{(0)}} \bar{\theta}_{0}+\mathcal{O}\left(\delta^{4}\right), \quad \mathcal{B}=\frac{V_{3}^{(1)}}{V_{2}^{(0)}} \bar{\theta}_{0}+\mathcal{O}\left(\delta^{6}\right) .
$$

After vacuum alignment, the $V_{i}$ coefficients are now functions of $\bar{\theta}_{0}+\sqrt{6} \eta_{0} / F_{0}$. Further, the normalization of the kinetic terms in the Lagrangian (2.3) provides

$$
V_{1}(0)=V_{2}(0)=\frac{F_{\pi}^{2}}{4}, \quad V_{4}(0)=\frac{1}{12}\left(F_{0}^{2}-F_{\pi}^{2}\right)
$$


In principle, the coupling of the $\eta_{0}$ singlet is different from $F_{\pi}$ because the subgroup $\mathrm{U}(3)_{V}$ does not present a nonet symmetry. However, in the large $N_{c}$-limit $F_{0}=F_{\pi}$. Moreover, the quantity of $\bar{\theta}_{0}$ can be denoted in terms of physical quantities [21]

$$
\bar{\theta}_{0}=\left[1+\frac{4 V_{0}^{(2)}}{F_{\pi}^{2}} \frac{4 M_{K}^{2}-M_{\pi}^{2}}{M_{\pi}^{2}\left(2 M_{K}^{2}-M_{\pi}^{2}\right)}\right]^{-1} \theta_{0} .
$$

Here, we note that $\bar{\theta}_{0}=\mathcal{O}\left(\delta^{2}\right)$, and take $1 / N_{c}=\mathcal{O}\left(\delta^{2}\right)$ as counting rules [19]. More detail and information on the formalism used in the work can be found in e.g. in refs. $[18,20]$.

We now turn to the baryon sector of the effective Lagrangian. In the SU(3) flavor representation the spin-1/2 anti-symmetric triplet and symmetric sextet charmed baryon states are denoted as in the following matrices, respectively,

$$
B_{\overline{3}}=\left(\begin{array}{ccc}
0 & \Lambda_{c}^{+} & \Xi_{c}^{+} \\
-\Lambda_{c}^{+} & 0 & \Xi_{c}^{0} \\
-\Xi_{c}^{+} & -\Xi_{c}^{0} & 0
\end{array}\right), \quad B_{6}=\left(\begin{array}{ccc}
\Sigma_{c}^{++} & \frac{\Sigma_{c}^{+}}{\sqrt{2}} & \frac{\Xi_{c}^{\prime}}{\sqrt{2}} \\
\frac{\Sigma_{c}^{+}}{\sqrt{2}} & \Sigma_{c}^{0} & \frac{\Xi_{c}^{\prime 0}}{\sqrt{2}} \\
\frac{\Xi_{c}^{\prime}}{\sqrt{2}} & \Xi_{c}^{\prime 0} & \Omega_{c}^{0}
\end{array}\right) .
$$

Similarly to the mesonic Lagrangian one can write down the most general effective Lagrangian for the charmed baryon multiplets. Here, we only present the terms pertinent to the calculation. In the quark mass and momentum expansion, the relevant free and interaction Lagrangians up to the second chiral order are given by [13, 15, 18, 22, 24],

$$
\begin{aligned}
\mathcal{L}_{\phi B, \text { free }}^{(1)}= & \frac{1}{2}\left\langle\bar{B}_{\overline{3}}\left(i \not D-m_{\overline{3}}\right) B_{\overline{3}}\right\rangle+\left\langle\bar{B}_{6}\left(i \not D-m_{6}\right) B_{\overline{6}}\right\rangle, \\
\mathcal{L}_{\phi B, \text { int }}^{(1)}= & \frac{g_{1}}{2}\left\langle\bar{B}_{6} \psi \gamma_{5} B_{6}\right\rangle+\frac{g_{2}}{2}\left[\left\langle\bar{B}_{6} \psi \gamma_{5} B_{\overline{3}}\right\rangle+h . c .\right]+\frac{g_{6}}{2}\left\langle\bar{B}_{\overline{3}} \psi \gamma_{5} B_{\overline{3}}\right\rangle \\
& +\frac{g_{1}}{2}\left\langle\bar{B}_{6} \gamma^{\mu} \gamma_{5} B_{6}\right\rangle\left\langle u_{\mu}\right\rangle+\frac{g_{2}}{2}\left[\left\langle\bar{B}_{6} \gamma^{\mu} \gamma_{5} B_{\overline{3}}\right\rangle+h . c .\right]\left\langle u_{\mu}\right\rangle+\frac{g_{6}}{2}\left\langle\bar{B}_{\overline{3}} \gamma^{\mu} \gamma_{5} B_{\overline{3}}\right\rangle\left\langle u_{\mu}\right\rangle, \\
\mathcal{L}_{\overline{3} \overline{3}}^{(2)}= & w_{16 / 17}\left\langle\bar{B}_{\overline{3}} \sigma^{\mu \nu} F_{\mu \nu}^{+} B_{\overline{3}}\right\rangle+w_{18}\left\langle\bar{B}_{\overline{3}} \sigma^{\mu \nu} B_{\overline{3}}\right\rangle\left\langle F_{\mu \nu}^{+}\right\rangle+b_{D / F}\left\langle\bar{B}_{\overline{3}} \tilde{\chi}_{+} B_{\overline{3}}\right\rangle+b_{0}\left\langle\bar{B}_{\overline{3}} B_{\overline{3}}\right\rangle\left\langle\tilde{\chi}_{+}\right\rangle \\
& +i w_{10 / 11} \frac{\sqrt{6}}{F_{0}} \eta_{0}\left\langle\bar{B}_{\overline{3}} \tilde{\chi}_{-} B_{\overline{3}}\right\rangle+i w_{12} \frac{\sqrt{6}}{F_{0}} \eta_{0}\left\langle\bar{B}_{\overline{3}} B_{\overline{3}}\right\rangle\left\langle\tilde{\chi}_{-}\right\rangle \\
& +i\left(w_{13 / 14}^{\prime} \bar{\theta}_{0}+w_{13 / 14} \frac{\sqrt{6}}{F_{0}} \eta_{0}\right)\left\langle\bar{B}_{\overline{3}} \sigma^{\mu \nu} \gamma_{5} F_{\mu \nu}^{+} B_{\overline{3}}\right\rangle \\
& +i\left(w_{15}^{\prime} \bar{\theta}_{0}+w_{15} \frac{\sqrt{6}}{F_{0}} \eta_{0}\right)\left\langle\bar{B}_{\overline{3}} \sigma^{\mu \nu} \gamma_{5} B_{\overline{3}}\right\rangle\left\langle F_{\mu \nu}^{+}\right\rangle, \\
\mathcal{L}_{66}^{(2)}= & w_{16 / 17}\left\langle\bar{B}_{6} \sigma^{\mu \nu} F_{\mu \nu}^{+} B_{6}\right\rangle+w_{18}\left\langle\bar{B}_{6} \sigma^{\mu \nu} B_{6}\right\rangle\left\langle F_{\mu \nu}^{+}\right\rangle+b_{D / F}\left\langle\bar{B}_{6} \tilde{\chi}_{+} B_{6}\right\rangle+b_{0}\left\langle\bar{B}_{6} B_{6}\right\rangle\left\langle\tilde{\chi}_{+}\right\rangle \\
& +i w_{10 / 11} \frac{\sqrt{6}}{F_{0}} \eta_{0}\left\langle\bar{B}_{6} \tilde{\chi}-B_{6}\right\rangle+i w_{12} \frac{\sqrt{6}}{F_{0}} \eta_{0}\left\langle\bar{B}_{6} B_{6}\right\rangle\left\langle\tilde{\chi}_{-}\right\rangle \\
& +i\left(w_{13 / 14}^{\prime} \bar{\theta}_{0}+w_{13 / 14} \frac{\sqrt{6}}{F_{0}} \eta_{0}\right)\left\langle\bar{B}_{6} \sigma^{\mu \nu} \gamma_{5} F_{\mu \nu}^{+} B_{6}\right\rangle \\
& +i\left(w_{15}^{\prime} \bar{\theta}_{0}+w_{15} \frac{\sqrt{6}}{F_{0}} \eta_{0}\right)\left\langle\bar{B}_{6} \sigma^{\mu \nu} \gamma_{5} B_{6}\right\rangle\left\langle F_{\mu \nu}^{+}\right\rangle, \\
\mathcal{L}_{6 \overline{3}}^{(2)}= & w_{16 / 17}\left\langle\bar{B}_{6} \sigma^{\mu \nu} F_{\mu \nu}^{+} B_{\overline{3}}\right\rangle+b_{D / F}\left\langle\bar{B}_{6} \tilde{\chi}_{+} B_{\overline{3}}\right\rangle+i w_{10 / 11} \frac{\sqrt{6}}{F_{0}} \eta_{0}\left\langle\bar{B}_{6} \tilde{\chi}_{-} B_{\overline{3}}\right\rangle \\
& +i w_{13 / 14} \frac{\sqrt{6}}{F_{0}} \eta_{0}\left\langle\bar{B}_{6} \sigma^{\mu \nu} \gamma_{5} F_{\mu \nu}^{+} B_{\overline{3}}\right\rangle+h . c .,
\end{aligned}
$$


where the relevant building blocks are

$$
\begin{aligned}
\tilde{\chi}_{-} & =\chi_{-}-i \mathcal{A}\left(U+U^{\dagger}\right)-i \mathcal{B} \chi_{+}, \\
\tilde{\chi}_{+} & =\chi_{+}-i \mathcal{A}\left(U-U^{\dagger}\right)-i \mathcal{B} \chi_{-}, \\
D_{\mu} B & =\partial_{\mu} B+\Gamma_{\mu} B+B \Gamma_{\mu}^{T}, \\
\Gamma_{\mu} & =\frac{1}{2}\left[u^{\dagger}\left(\partial \mu-i r_{\mu}\right) u+u\left(\partial \mu-i l_{\mu}\right) u^{\dagger}\right], \\
u & =i\left[u^{\dagger}\left(\partial \mu-i r_{\mu}\right) u-u\left(\partial \mu-i l_{\mu}\right) u^{\dagger}\right] .
\end{aligned}
$$

The charge matrix for the singly-charmed baryons is $Q_{h}=\operatorname{diag}(1,0,0)$, while for the light quarks the charge matrix is $Q_{l}=\operatorname{diag}(2 / 3,-1 / 3,-1 / 3)$. We use $w_{10 / 11}+3 w_{12}=w_{10}^{\prime}$ as in ref. [21].

As can be seen from the contributing Lagrangians, there are quite number of low-energy constants (LECs). The meson-baryon coupling constants $g_{i}(i=1, \ldots, 6)$, the symmetrybreaking LECs $b_{D}$ and $b_{F}$ as well as the LECs $w_{16 / 17}, w_{18}$ related to the CP-conserving electromagnetic response can all be taken from earlier studies of different observables, as detailed in section 4 .

This leaves us with the yet undetermined LECs $w_{10}^{\prime}, w_{13 / 14}^{\prime}, w_{15}^{\prime}$ and $w_{13 / 14}, w_{15}$. As will be shown, we can fix $w_{13 / 14}, w_{15}$ from recent lattice results QCD for the neutron and proton electric dipole moments, $d_{n}$ and $d_{p}$, respectively. The remaining of these LECs will be varied as $0_{-0.5}^{+0.5} \mathrm{GeV}^{-1}$, that is within a natural range. This naive dimensional analysis should be eventually overcome by a more sophisticated modeling of the LECs or invoking further lattice QCD results. Having fixed/estimated all the LECs will then allow to estimate the $\mathrm{CP}$-violating contributions to the singly-charmed baryons induced by the $\theta$-term.

\section{CP-violating electromagnetic form factor}

The electromagnetic form factors of a baryon are defined via the matrix element of the electromagnetic current,

$$
\begin{aligned}
\left\langle B\left(p_{f}\right)\left|J_{\mathrm{em}}^{\mu}\right| B\left(p_{i}\right)\right\rangle=\bar{u}\left(p_{f}\right) & {\left[\gamma^{\mu} F_{1}\left(q^{2}\right)-\frac{i F_{2}\left(q^{2}\right)}{2 m_{B}} \sigma^{\mu \nu} q_{\nu}\right.} \\
& \left.+i\left(\gamma^{\mu} q^{2} \gamma_{5}-2 m_{B} q^{\mu} \gamma_{5}\right) F_{A}\left(q^{2}\right)-\frac{F_{3}\left(q^{2}\right)}{2 m_{B}} \sigma^{\mu \nu} q_{\nu} \gamma_{5}\right] u\left(p_{i}\right)
\end{aligned}
$$

with $q^{2}=\left(p_{f}-p_{i}\right)^{2}$ the invariant momentum transfer squared, $m_{B}$ the baryon mass and $J_{\mathrm{em}}^{\mu}$ the electromagnetic current. Here, $F_{1}\left(q^{2}\right)$ and $F_{2}\left(q^{2}\right)$ are the P- and CP-conserving Dirac and Pauli form factors, respectively. $F_{A}\left(q^{2}\right)$ denotes the P-violating anapole form factor, and $F_{3}\left(q^{2}\right)$, which will be considered throughout this work, the P- and CP-violating electric dipole form factor. The electric dipole moment of the baryon $B$ is then given by

$$
d_{B}=\frac{F_{3, B}(0)}{2 m_{B}}
$$

In what follows, we will use the effective Lagrangian to calculate the CP-violating form factor of the singly-charmed baryons at next-to-leading (NLO) order, which includes tree as well as loop diagrams as shown in figure 1, where we display the corresponding 
Feynman diagrams. Tree-level diagrams at leading order are presented in (a) and (b). One-loop diagrams at order $\mathcal{O}\left(\delta^{2}\right)$ and $\mathcal{O}\left(\delta^{3}\right)$ in (c)-(d), and (e)-(h), respectively. The type of diagrams in (g)-(h) with pionic or kaonic loops of the antitriplet and the sextet charmed baryons are canceling each other, thus they are not displayed here.

We show different combinations of the charmed baryon states from anti-triplet and sextet multiplets considered throughout the calculation in figure 2 .

The results obtained for the form factor $F_{3}\left(q^{2}\right)$ of the charmed baryons coming from the tree-level diagrams are collected in table 1 with

$$
\alpha=\frac{576 V_{0}^{(2)} V_{3}^{(1)}}{\left(F_{0} F_{\pi} M_{\eta_{0}}\right)^{2}} .
$$

As usual in the EOMS scheme, the loop contributions are rather lengthy expression. Let us discuss the case of the $\Lambda_{c}^{+}$. The one-loop contribution can be written as, cf. figure 1 ,

$$
\begin{aligned}
& F_{3, \Lambda_{c}^{+}}^{\text {loop }}\left(q^{2}\right)=\sum_{i=1}^{2} \frac{e \bar{\theta}_{0} V_{0}^{(2)} \tilde{m}}{\pi^{2} F_{\pi}^{4}} \frac{1}{\left(4 \tilde{m}^{2}-q^{2}\right)} \\
& \times\left[C _ { c d } ^ { i } ( m _ { i } ( \tilde { m } + m _ { i } ) ) \left(2 J_{i}^{c d}\left(\tilde{m}^{2}, m_{i}^{2}, M^{2}\right)-2 J_{i}^{c d}\left(q^{2}, M^{2}, M^{2}\right)\right.\right. \\
& \left.\left.+\left(2 M^{2}+2 \tilde{m}^{2}-2 m_{i}^{2}-q^{2}\right) J_{i}^{c d}\left(\tilde{m}^{2}, \tilde{m}^{2}, q^{2}, M^{2}, m_{i}^{2}, M^{2}\right)\right)\right] \\
& +\sum_{i=3}^{4} \frac{e \bar{\theta}_{0} V_{0}^{(2)}}{\pi^{2} F_{\pi}^{4}} \frac{1}{\left(4 \tilde{m}^{2}-q^{2}\right)}\left[C _ { e f } ^ { i } \left(-J_{i}^{e f}\left(M_{i}^{2}\right)\left(4 \tilde{m}^{2}-q^{2}\right)+J_{i}^{e f}\left(\tilde{m}^{2}\right)\left(4 \tilde{m}^{2}-q^{2}\right)\right.\right. \\
& -\left(M_{i}^{2}\left(q^{2}+4 \tilde{m}^{2}\right)-4 \tilde{m}^{2} q^{2}\right) J_{i}^{e f}\left(\tilde{m}^{2}, \tilde{m}^{2}, M_{i}^{2}\right)+8 \tilde{m}^{2}\left(M_{i}^{2}-2 \tilde{m}^{2}\right) J_{i}^{e f}\left(q^{2}, \tilde{m}^{2}, \tilde{m}^{2}\right) \\
& \left.\left.+4 \tilde{m}^{2} M_{i}^{2}\left(2 M_{i}^{2}-8 \tilde{m}^{2}+q^{2}\right) J_{i}^{e f}\left(\tilde{m}^{2}, \tilde{m}^{2}, q^{2}, \tilde{m}^{2}, M^{2}, \tilde{m}^{2}\right)\right)\right] \\
& +\sum_{i=5}^{7} \frac{e \bar{\theta}_{0} V_{0}^{(2)}}{\pi^{2} F_{\pi}^{4}} \frac{1}{\left(4 \tilde{m}^{2}-q^{2}\right)}\left[C _ { e f } ^ { i } \left(-J_{i}^{e f}\left(M_{i}^{2}\right)\left(4 \tilde{m}^{2}-q^{2}\right)+J_{i}^{e f}\left(m_{i}^{2}\right)\left(4 \tilde{m}^{2}-q^{2}\right)\right.\right. \\
& -\left(M_{i}^{2}\left(4 \tilde{m} m_{i}+q^{2}\right)+\left(m \tilde{+} m_{i}\right)\left(4 \tilde{m}^{2} m_{i}-m_{i} q^{2}-\tilde{m}\left(4 m_{i}^{2}+q^{2}\right)\right)\right) J_{i}^{e f}\left(\tilde{m}^{2}, m_{i}^{2}, M_{i}^{2}\right) \\
& -4 \tilde{m}\left(\tilde{m}+m_{i}\right)\left(\tilde{m}^{2}+m_{i}^{2}-M_{i}^{2}\right) J_{i}^{e f}\left(q^{2}, m_{i}^{2}, m_{i}^{2}\right)+2 \tilde{m}\left(\tilde{m}+m_{i}\right) \\
& \times\left(2 M_{i}^{4}+\left(\tilde{m}^{2}-m_{i}^{2}\right)\left(2 \tilde{m}^{2}-2 m_{i}^{2}-q^{2}\right)\right. \\
& \left.\left.\left.+M_{i}^{2}\left(q^{2}-4 \tilde{m}^{2}-4 m_{i}^{2}\right)\right) J_{i}^{e f}\left(\tilde{m}^{2}, \tilde{m}^{2}, q^{2}, m_{i}^{2}, M_{i}^{2}, m_{i}^{2}\right)\right)\right] \\
& +\sum_{i=8}^{10} \frac{e \bar{\theta}_{0} V_{0}^{(2)}}{\pi^{2} F_{\pi}^{4}} \frac{1}{\left(4 \tilde{m}^{2}-q^{2}\right)}\left[C _ { e f } ^ { i } \left(-J_{i}^{e f}\left(M_{i}^{2}\right)\left(4 \tilde{m}^{2}-q^{2}\right)+J_{i}^{e f}\left(m_{i}^{2}\right)\left(4 \tilde{m}^{2}-q^{2}\right)\right.\right. \\
& +\left(\tilde{m}^{2} q^{2}-4 \tilde{m}^{3} m_{i}+q^{2}\left(m_{i}^{2}-M_{i}^{2}\right)+2 \tilde{m} m_{i}\left(q^{2}+2 m_{i}^{2}-2 M_{i}^{2}\right)\right) J_{i}^{e f}\left(\tilde{m}^{2}, m_{i}^{2}, M_{i}^{2}\right) \\
& -4 \tilde{m}\left(\tilde{m}+m_{i}\right)\left(\tilde{m}^{2}+m_{i}^{2}-M_{i}^{2}\right) J_{i}^{e f}\left(q^{2}, m_{i}^{2}, m_{i}^{2}\right)+2 \tilde{m}\left(\tilde{m}+m_{i}\right) \\
& \times\left(2 M_{i}^{4}+2 \tilde{m}^{4}+M_{i}^{2}\left(q^{2}-4 m_{i}^{2}\right)+m_{i}^{2}\left(2 m_{i}^{2}+q^{2}\right)\right. \\
& \left.\left.\left.\left.-\tilde{m}^{2}\left(4 M_{i}^{2}+4 m_{i}^{2}+q^{2}\right)\right)\right) J_{i}^{e f}\left(\tilde{m}^{2}, \tilde{m}^{2}, q^{2}, m_{i}^{2}, M_{i}^{2}, m_{i}^{2}\right)\right)\right] \\
& +\frac{16 e \bar{\theta}_{0} V_{0}^{(2)}}{\pi^{2} F_{\pi}^{2} F_{0}^{2}}\left[C_{g h}^{11}\left(J_{11}^{g h}\left(M^{2}\right)-J_{11}^{g h}\left(\tilde{m}^{2}\right)-\left(M^{2}-4 \tilde{m}^{2}\right) J_{11}^{g h}\left(\tilde{m}^{2}, M^{2}\right)\right)\right],
\end{aligned}
$$




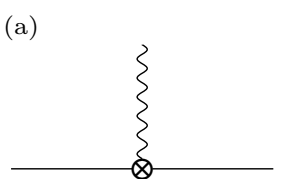

(b)

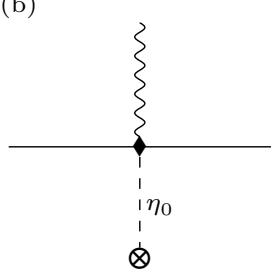

(f)
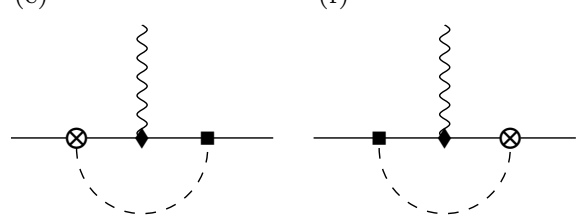

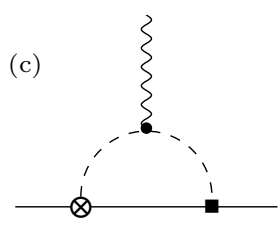

(d)

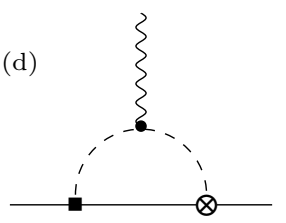

(g)
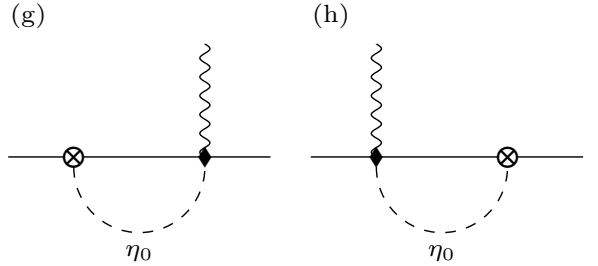

Figure 1. CP-violating contributions of the spin- $1 / 2$ charmed baryons. Solid lines correspond to contribution from either spin- $1 / 2$ anti-triplet or sextet multiplets of charmed baryons. Filled circles are second-order mesonic vertices, squares and diamonds represent vertices generated by the first and second order meson-baryon Lagrangian, respectively. CP-violating vertices are denoted by $\otimes$.
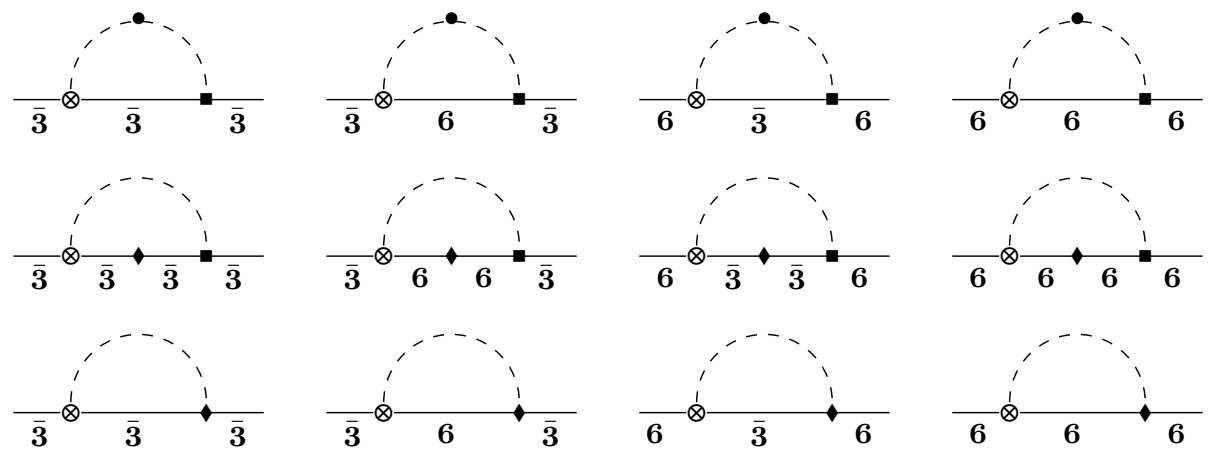

Figure 2. Different combinations of the spin- $1 / 2$ anti-triplet and sextet charmed baryons contributing to $F_{3}\left(q^{2}\right)$.

with $m_{i}, \tilde{m}_{i}$ and $M_{i}$ denoting the masses of the corresponding internal and external baryons and meson running in the loop, for notational simplicity. In the case at hand, $\tilde{m}=$ $m_{\Lambda_{c}^{+}}$. The $J\left(m_{i}, M_{i}, q^{2}\right)$ functions can be reduced to the scalar loop functions given in appendix A, and the labels $c d$, ef and $g h$ refer to the types of diagrams shown in figure 1. The corresponding coefficients $C_{c d}, C_{e f}$ and $C_{g h}$ for the $\Lambda_{c}^{+}$together with the intermediate meson-baryon states are shown in table 2, the corresponding tables for the other particles can be found in appendix B. A MATHEMaticA notebook with these loop functions can be obtained from the first author of this paper. 


\begin{tabular}{|cll|}
\hline States & Contributions \\
\hline & $\Lambda_{c}^{+}$ & $e \bar{\theta}_{0} m_{\Lambda_{c}}\left[2 \alpha\left(w_{13 / 14}+2 w_{15}\right)+8\left(w_{13 / 14}^{\prime}+2 w_{15}^{\prime}\right)\right]$ \\
$B_{\overline{3}}$ & $\Xi_{c}^{+}$ & $e \bar{\theta}_{0} m_{\Xi_{c}}\left[2 \alpha\left(w_{13 / 14}+2 w_{15}\right)+8\left(w_{13 / 14}^{\prime}+2 w_{15}^{\prime}\right)\right]$ \\
& $\Xi_{c}^{0}$ & $e \bar{\theta}_{0} m_{\Xi_{c}} 4\left(\alpha w_{15}+4 w_{15}^{\prime}\right)$ \\
\hline & $\Sigma_{c}^{++}$ & $e \bar{\theta}_{0} m_{\Sigma_{c}}\left[2 \alpha\left(w_{13 / 14}+w_{15}\right)+8\left(w_{13 / 14}^{\prime}+w_{15}^{\prime}\right)\right]$ \\
& $\Sigma_{c}^{+}$ & $e \bar{\theta}_{0} m_{\Sigma_{c}}\left[\alpha\left(w_{13 / 14}+2 w_{15}\right)+4\left(w_{13 / 14}^{\prime}+2 w_{15}^{\prime}\right)\right]$ \\
& $\Sigma_{c}^{0}$ & $e \bar{\theta}_{0} m_{\Sigma_{c}} 2\left(\alpha w_{15}+4 w_{15}^{\prime}\right)$ \\
$B_{6}$ & $\Xi_{c}^{\prime+}$ & $e \bar{\theta}_{0} m_{\Xi_{c}^{\prime}}\left[\alpha\left(w_{13 / 14}+2 w_{15}\right)+4\left(w_{13 / 14}^{\prime}+2 w_{15}^{\prime}\right)\right]$ \\
& $\Xi_{c}^{\prime}$ & $e \bar{\theta}_{0} m_{\Xi_{c}^{\prime}} 2\left(\alpha w_{15}+4 w_{15}^{\prime}\right)$ \\
& $\Omega_{c}^{0}$ & $e \bar{\theta}_{0} m_{\Omega_{c}} 2\left(\alpha w_{15}+4 w_{15}^{\prime}\right)$ \\
\hline
\end{tabular}

Table 1. Tree-level contribution to the $F_{3}\left(q^{2}\right)$ of the charmed baryons.

\begin{tabular}{|cccc|}
\hline Diagram type & number & meson-baryon state & Coefficient \\
\hline (c), (d) & 1 & $\Xi_{c}^{0}, K^{ \pm}$ & $2 g_{6} b_{D / F}$ \\
& 2 & $\Xi_{c}^{\prime 0}, K^{ \pm}$ & $g_{2} b_{D / F}$ \\
\hline & 3 & $\Lambda_{c}^{+}, \eta_{8}$ & $\frac{8}{3} g_{6} b_{D / F}\left(w_{16 / 17}+2 w_{18}\right)$ \\
& 4 & $\Lambda_{c}^{+}, \eta_{0}$ & $\frac{32}{3} \beta g_{6}\left(w_{16 / 17}+2 w_{18}\right)$ \\
& 5 & $\Xi_{c}^{+}, K^{0}$ & $4 g_{6} b_{D / F}\left(w_{16 / 17}+2 w_{18}\right)$ \\
$(\mathrm{e}),(\mathrm{f})$ & 6 & $\Xi_{c}^{\prime}, K^{ \pm}$ & $2 g_{2} b_{D / F} w_{18}$ \\
& 7 & $\Xi_{c}^{+}, K^{0}$ & $g_{2} b_{D / F}\left(w_{16 / 17}+2 w_{18}\right)$ \\
& 8 & $\Sigma_{c}^{0}, \pi^{ \pm}$ & $4 g_{2} b_{D / F} w_{18}$ \\
& 9 & $\Sigma_{c}^{+}, \pi^{0}$ & $2 g_{2} b_{D / F}\left(w_{16 / 17}+2 w_{18}\right)$ \\
& 10 & $\Sigma_{c}^{++}, \pi^{ \pm}$ & $4 g_{2} b_{D / F}\left(w_{16 / 17}+w_{18}\right)$ \\
\hline$(\mathrm{g}),(\mathrm{h})$ & 11 & $\Lambda_{c}^{+}, \eta_{0}$ & $\beta\left(w_{13 / 14}+2 w_{15}\right)$ \\
\hline
\end{tabular}

Table 2. Loop contribution to the $F_{3}\left(q^{2}\right)$ of the $\Lambda_{c}^{+}$baryon with $\beta=\left(b_{D / F}+b_{0}+3 w^{\prime}{ }_{10}\right)$. 


\section{Results}

First, we must fix parameters. The pion decay constant is taken as $F_{\pi}=92.2 \mathrm{MeV}$. In what follows, due to the lack of data from the charmed meson sector, we make recourse to the ground state baryon octet as much as possible to fix as many LECs as possible. While this is an approximation, we expect that we are estimating at least the right order of magnitude of the EDMs of the charmed baryons. Consequently, two symmetry-breaking LECs in the baryon sector can be obtained from baryon mass splittings. We use $b_{D}=-0.606 \mathrm{GeV}^{-1}$ and $b_{F}=-0.209 \mathrm{GeV}^{-1}[23,24]$. The tree-level contributions can be expressed in terms of two independent linear combinations of unknown LECs as $\alpha\left(w_{13 / 14}+4 w_{13 / 14}^{\prime}\right)$ and $\alpha w_{15}+4 w_{15}^{\prime}$, cf. table 1. The loop contributions are also dependent on unknown LECs, viz., $w_{10}^{\prime}, w_{13 / 14}$ and $w_{15}$. The conventional magnetic moment couplings, $w_{18}$ is taken equal to $w_{16 / 17}=0.40$, determined from fits to calculations to baryon magnetic moments in $[25,26]$.

Further, $V_{0}^{(2)}=-5 \times 10^{-4} \mathrm{GeV}^{4}$ and $V_{3}^{(1)}=3.5 \times 10^{-4} \mathrm{GeV}^{2}$ are the values obtained from an analysis of $\eta-\eta^{\prime}$ mixing in $\mathrm{U}(3)$ chiral perturbation theory [27]. The various baryon-meson couplings are taken from refs. $[13,14], g_{1}=0.98, g_{2}=-0.60, g_{3}=0.85$, and $g_{4}=1.04$. Because of the forbidden $B_{\overline{3}} B_{\overline{3}} \phi$-vertex, we have $g_{6}=0$. We use the physical masses of the pertinent mesons and baryons running in the corresponding loops, cf. tables $4-11$.

As the unknown LECs cannot be parameterized such a common constant as in [24], since the combinations coming from different particles are different, they have to be considered individually. Using the lattice data from [28] at physical pion mass, we use the neutron dipole moment to fix $\beta w_{15}$ from the $\Xi_{c}^{0}$ by comparing the loop contributions. With that, we can use the proton electric dipole moment to determine $\beta w_{13 / 14}$ from the $\Lambda_{c}^{+}$. We get

$$
\beta w_{13 / 14}=-0.00435 \mathrm{GeV}^{-1}, \quad \beta w_{15}=0.00175 \mathrm{GeV}^{-1} .
$$

With these obtained values, we take the variation of $w_{10}^{\prime}, w_{13 / 14}^{\prime}$ and $w_{15}^{\prime}$, and calculate the CP-violating form factor $F_{3}\left(q^{2}\right)$ for the singly-charmed baryons in the range $q^{2} \simeq 0.05 \ldots 0.3 \mathrm{GeV}^{2}$ as given in tables $12-14$. We are well aware that there are other determinations of $d_{p}$ and $d_{n}$ in the literature, see e.g. refs. [29, 30], and that there is an on-going debate on the axial rotation in the finite volume (mixing between the form factors $F_{2}$ and $F_{3}$, see e.g. ref. [31]). However, since our study is largely exploratory, we do not explore the whole possible parameter space.

The electric dipole moments for the various baryons are collected in table 3. As there is a sizeable uncertainty induced by the unknown LECs, we refrain from performing a systematic error analysis accounting e.g. for the effects of higher orders in the chiral expansion. Hopefully, lattice QCD will be able to supply pertinent information on the LECs so that more accurate predictions can be made.

\section{Conclusion}

In this paper, we have performed a one-loop calculation of the $\mathrm{CP}$-violating form factor $F_{3}\left(q^{2}\right)$ and the corresponding electric dipole moments of the spin- $1 / 2$ singly-charmed 


\begin{tabular}{|lccccccccc|}
\hline & $\Lambda_{c}^{+}$ & $\Xi_{c}^{+}$ & $\Xi_{c}^{0}$ & $\Sigma_{c}^{++}$ & $\Sigma_{c}^{+}$ & $\Sigma_{c}^{0}$ & $\Xi_{c}^{\prime+}$ & $\Xi_{c}^{\prime 0}$ & $\Omega_{c}^{0}$ \\
\hline Set 1 & 0.0476 & 0.0470 & 0.0294 & 0.0047 & 0.0053 & 0.0058 & 0.0007 & 0.0085 & 0.0067 \\
Set 2 & 0.0011 & 0.0005 & -0.0015 & -0.0090 & -0.0049 & -0.0010 & -0.0097 & 0.0015 & -0.0003 \\
Set 3 & -0.0454 & -0.0460 & -0.0324 & -0.0228 & -0.0153 & -0.0078 & -0.0202 & -0.0053 & -0.0074 \\
\hline
\end{tabular}

Table 3. Electric dipole moments for the singly-charmed baryons in units of $e \theta_{0} \mathrm{fm}$. Set $1,2,3$ refers to $w_{10}^{\prime}=w_{13 / 14}^{\prime}=w_{15}^{\prime}=-0.5,0,+0.5$, in order.

baryons, where the mechanism of the $\mathrm{CP}$ violation is the QCD $\theta$-term. Not all the appearing low-energy constants could be fixed from experimental or lattice QCD data, so the resulting predictions show a spread, cf. table 3 and the tables in appendix C. We hope that with more lattice QCD studies on strong CP violations, these LECs can be determined and more accurate predictions can be made, not to mention possible experimental determinations.

\section{Acknowledgments}

We thank Jambul Gegelia, Sebastian Neubert, Altuğ Özpineci and Daniel Severt for discussions. Partial financial support from the Deutsche Forschungsgemeinschaft (Project-ID 196253076 - TRR 110, "Symmetries and the Emergence of Structure in QCD"), by the Chinese Academy of Sciences (CAS) President's International Fellowship Initiative (PIFI) (grant no. 2018DM0034), by VolkswagenStiftung (grant no. 93562) and by the EU (Strong2020) is acknowledged.

\section{A Scalar loop integrals}

The scalar loop integrals of one-, two-, and three-point functions which are used for the calculation of the diagrams are given by

$$
\begin{aligned}
J_{0}\left(m^{2}\right) & =\frac{(2 \pi \mu)^{4-d}}{i \pi^{2}} \int \frac{d^{d} k}{k^{2}-m^{2}+i 0^{+}}, \\
J_{0}\left(p^{2}, m_{1}^{2}, m_{2}^{2}\right) & =\frac{(2 \pi \mu)^{4-d}}{i \pi^{2}} \int \frac{d^{d} k}{\left[k^{2}-m_{1}^{2}+i 0^{+}\right]\left[(k+p)^{2}-m_{2}^{2}+i 0^{+}\right]}, \\
J_{0}\left(p_{i}^{2},\left(p_{f}-p_{i}\right)^{2},\right. & \left.p_{f}^{2}, m_{1}^{2}, m_{2}^{2}, m_{3}^{2}\right) \\
& =\frac{(2 \pi \mu)^{4-d}}{i \pi^{2}} \int \frac{d^{d} k}{\left[k^{2}-m_{1}^{2}+i 0^{+}\right]\left[\left(k-p_{i}\right)^{2}-m_{2}^{2}+i 0^{+}\right]\left[\left(k-p_{f}\right)^{2}-m_{3}^{2}+i 0^{+}\right]} .
\end{aligned}
$$

\section{B Loop contributions}

All one-loop contributions to the various baryons take the form as given in eq. (3.4). In this appendix, we collect the corresponding intermediate meson-baryon states and the values of the coefficients $C^{c d}, C^{e f}$ and $C^{g h}$ for the baryons not given in the main text. 


\begin{tabular}{|cccc|}
\hline Diagram type & Number & Meson-baryon state & Coefficient \\
\hline (c), (d) & 1 & $\Xi_{c}^{0}, \pi^{ \pm}$ & $g_{6} b_{D / F}$ \\
& 2 & $\Omega_{c}^{0}, K^{ \pm}$ & $g_{2} b_{D / F}$ \\
& 3 & $\Sigma_{c}^{++}, K^{ \pm}$ & $g_{2} b_{D / F}$ \\
& 4 & $\Xi_{c}^{\prime 0}, \pi^{ \pm}$ & $g_{2} b_{D / F}$ \\
\hline & 5 & $\Xi_{c}^{+}, \eta_{8}$ & $g_{6} b_{D / F}\left(w_{16 / 17}+2 w_{18}\right)$ \\
& 6 & $\Xi_{c}^{+}, \eta_{0}$ & $\beta g_{6}\left(w_{16 / 17}+2 w_{18}\right)$ \\
& 7 & $\Xi_{c}^{+}, \pi^{0}$ & $g_{6} b_{D / F}\left(w_{16 / 17}+2 w_{18}\right)$ \\
& 8 & $\Xi_{c}^{\prime 0}, \pi^{ \pm}$ & $g_{2} b_{D / F} w_{18}$ \\
$(\mathrm{e}),(\mathrm{f})$ & 9 & $\Xi_{c}^{+}, \eta_{8}$ & $g_{2} b_{D / F}\left(w_{16 / 17}+2 w_{18}\right)$ \\
& 10 & $\Sigma_{c}^{+}, K^{0}$ & $g_{2} b_{D / F}\left(w_{16 / 17}+2 w_{18}\right)$ \\
& 11 & $\Xi_{c}^{\prime}, \pi^{0}$ & $g_{2} b_{D / F}\left(w_{16 / 17}+2 w_{18}\right)$ \\
& 12 & $\Sigma_{c}^{++}, K^{ \pm}$ & $g_{2} b_{D / F}\left(w_{16 / 17}+w_{18}\right)$ \\
& 13 & $\Omega_{c}^{0}, K^{ \pm}$ & $g_{2} b_{D / F} w_{18}$ \\
\hline (g), (h) & 14 & $\Xi_{c}^{+}, \eta_{0}$ & $\beta\left(w_{13 / 14}+2 w_{15}\right)$ \\
\hline
\end{tabular}

Table 4. Loop contribution to the $F_{3}\left(q^{2}\right)$ of the $\Xi_{c}^{+}$baryon.

\begin{tabular}{|cccc|}
\hline Diagram type & Number & Meson-baryon state & Coefficient \\
\hline (c), (d) & 1 & $\Lambda_{c}^{+}, K^{ \pm}$ & $g_{6} b_{D / F}$ \\
& 2 & $\Omega_{c}^{0}, K^{ \pm}$ & $g_{2} b_{D / F}$ \\
& 3 & $\Xi_{c}^{+}, \pi^{ \pm}$ & $g_{6} b_{D / F}$ \\
& 4 & $\Sigma_{c}^{+}, K^{ \pm}$ & $g_{2} b_{D / F}$ \\
& 5 & $\Xi_{c}^{\prime}, \pi^{ \pm}$ & $g_{2} b_{D / F}$ \\
\hline & 6 & $\Xi_{c}^{0}, \eta_{8}$ & $g_{6} b_{D / F} w_{18}$ \\
& 7 & $\Xi_{c}^{0}, \eta_{0}$ & $\beta g_{6} w_{18}$ \\
& 8 & $\Xi_{c}^{0}, \pi^{0}$ & $g_{6} b_{D / F} w_{18}$ \\
& 9 & $\Xi_{c}^{\prime}, \pi^{ \pm}$ & $g_{2} b_{D / F}\left(w_{16 / 17}+2 w_{18}\right)$ \\
& 10 & $\Sigma_{c}^{0}, K^{0}$ & $g_{2} b_{D / F} w_{18}$ \\
& 11 & $\Xi_{c}^{\prime}, \eta_{8}$ & $g_{2} b_{D / F} w_{18}$ \\
& 12 & $\Xi_{c}^{\prime 0}, \pi^{0}$ & $g_{2} b_{D / F} w_{18}$ \\
& 13 & $\Sigma_{c}^{+}, K^{ \pm}$ & $g_{2} b_{D / F}\left(w_{16 / 17}+2 w_{18}\right)$ \\
& 14 & $\Omega_{c}^{0}, K^{0}$ & $g_{2} b_{D / F} w_{18}$ \\
\hline (g), (h) & 15 & $\Xi_{c}^{0}, \eta_{0}$ & $\beta w_{15}$ \\
\hline
\end{tabular}

Table 5. Loop contribution to the $F_{3}\left(q^{2}\right)$ of the $\Xi_{c}^{0}$ baryon. 


\begin{tabular}{|cccc|}
\hline Diagram type & Number & Meson-baryon state & Coefficient \\
\hline & 1 & $\Xi_{c}^{\prime}, K^{ \pm}$ & $g_{1} b_{D / F}$ \\
$(\mathrm{c}),(\mathrm{d})$ & 2 & $\Sigma_{c}^{+}, \pi^{ \pm}$ & $g_{1} b_{D / F}$ \\
& 3 & $\Xi_{c}^{+}, K^{ \pm}$ & $g_{2} b_{D / F}$ \\
& 4 & $\Lambda_{c}^{+}, \pi^{ \pm}$ & $g_{2} b_{D / F}$ \\
\hline & 5 & $\Sigma_{c}^{++}, \eta_{8}$ & $g_{1} b_{D / F}\left(w_{16 / 17}+w_{18}\right)$ \\
& 6 & $\Sigma_{c}^{++}, \eta_{0}$ & $\beta g_{1}\left(w_{16 / 17}+w_{18}\right)$ \\
$(\mathrm{e}),(\mathrm{f})$ & 7 & $\Sigma_{c}^{++}, \pi^{0}$ & $g_{1} b_{D / F}\left(w_{16 / 17}+w_{18}\right)$ \\
& 8 & $\Xi_{c}^{+}, K^{ \pm}$ & $g_{2} b_{D / F}\left(w_{16 / 17}+2 w_{18}\right)$ \\
& 9 & $\Lambda_{c}^{0}, \pi^{ \pm}$ & $g_{2} b_{D / F}\left(w_{16 / 17}+2 w_{18}\right)$ \\
\hline (g), (h) & 10 & $\Sigma_{c}^{++}, \eta_{0}$ & $\beta\left(w_{13 / 14}+w_{15}\right)$ \\
\hline
\end{tabular}

Table 6. Loop contribution to the $F_{3}\left(q^{2}\right)$ of the $\Sigma_{c}^{++}$baryon.

\begin{tabular}{|cccc|}
\hline Diagram type & Number & Meson-baryon state & Coefficient \\
\hline$(\mathrm{c}),(\mathrm{d})$ & 1 & $\Xi_{c}^{\prime 0}, K^{ \pm}$ & $g_{1} b_{D / F}$ \\
& 2 & $\Xi_{c}^{0}, K^{ \pm}$ & $g_{2} b_{D / F}$ \\
\hline & 3 & $\Sigma_{c}^{+}, \eta_{8}$ & $g_{1} b_{D / F}\left(w_{16 / 17}+2 w_{18}\right)$ \\
& 4 & $\Sigma_{c}^{+}, \eta_{0}$ & $\beta g_{1}\left(w_{16 / 17}+2 w_{18}\right)$ \\
$(\mathrm{e}),(\mathrm{f})$ & 5 & $\Xi_{c}^{+}, K^{0}$ & $g_{2} b_{D / F}\left(w_{16 / 17}+w_{18}\right)$ \\
& 6 & $\Xi_{c}^{0}, K^{ \pm}$ & $g_{2} b_{D / F} w_{18}$ \\
& 7 & $\Lambda_{c}^{0}, \pi^{0}$ & $g_{2} b_{D / F}\left(w_{16 / 17}+2 w_{18}\right)$ \\
\hline$(\mathrm{g}),(\mathrm{h})$ & 8 & $\Sigma_{c}^{+}, \eta_{0}$ & $\beta\left(w_{13 / 14}+2 w_{15}\right)$ \\
\hline
\end{tabular}

Table 7. Loop contribution to the $F_{3}\left(q^{2}\right)$ of the $\Sigma_{c}^{+}$baryon.

\begin{tabular}{|cccc|}
\hline Diagram type & Number & Meson-baryon state & Coefficient \\
\hline (c), (d) & 1 & $\Sigma_{c}^{+}, \pi^{ \pm}$ & $g_{1} b_{D / F}$ \\
& 2 & $\Lambda_{c}^{0}, \pi^{ \pm}$ & $g_{2} b_{D / F}$ \\
\hline & 3 & $\Sigma_{c}^{0}, \eta_{8}$ & $g_{1} b_{D / F} w_{18}$ \\
& 4 & $\Sigma_{c}^{0}, \eta_{0}$ & $\beta g_{1} w_{18}$ \\
$(\mathrm{e}),(\mathrm{f})$ & 5 & $\Sigma_{c}^{0}, \pi^{0}$ & $g_{1} b_{D / F} w_{18}$ \\
& 6 & $\Xi_{c}^{0}, K^{0}$ & $g_{2} b_{D / F} w_{18}$ \\
& 7 & $\Lambda_{c}^{0}, \pi^{ \pm}$ & $g_{2} b_{D / F}\left(w_{16 / 17}+2 w_{18}\right)$ \\
\hline$(\mathrm{g}),(\mathrm{h})$ & 8 & $\Sigma_{c}^{0}, \eta_{0}$ & $\beta w_{15}$ \\
\hline
\end{tabular}

Table 8. Loop contribution to the $F_{3}\left(q^{2}\right)$ of the $\Sigma_{c}^{0}$ baryon. 


\begin{tabular}{|cccc|}
\hline Diagram type & Number & Meson-baryon state & Coefficient \\
\hline & 1 & $\Omega_{c}^{0}, K^{ \pm}$ & $g_{1} b_{D / F}$ \\
$(\mathrm{c}),(\mathrm{d})$ & 2 & $\Sigma_{c}^{++}, K^{ \pm}$ & $g_{1} b_{D / F}$ \\
& 3 & $\Xi_{c}^{\prime}, \pi^{ \pm}$ & $g_{1} b_{D / F}$ \\
& 4 & $\Xi_{c}^{0}, \pi^{ \pm}$ & $g_{2} b_{D / F}$ \\
\hline & 5 & $\Xi_{c}^{+}, \eta_{8}$ & $g_{1} b_{D / F}\left(w_{16 / 17}+2 w_{18}\right)$ \\
& 6 & $\Xi_{c}^{+}, \eta_{0}$ & $\beta g_{1}\left(w_{16 / 17}+2 w_{18}\right)$ \\
& 7 & $\Xi_{c}^{+}, \pi^{0}$ & $g_{1} b_{D / F}\left(w_{16 / 17}+2 w_{18}\right)$ \\
$(\mathrm{e}),(\mathrm{f})$ & 8 & $\Sigma_{c}^{+}, K^{0}$ & $g_{1} b_{D / F}\left(w_{16 / 17}+2 w_{18}\right)$ \\
& 9 & $\Xi_{c}^{+}, \eta_{8}$ & $g_{2} b_{D / F}\left(w_{16 / 17}+2 w_{18}\right)$ \\
& 10 & $\Lambda_{c}^{+}, K^{0}$ & $g_{2} b_{D / F}\left(w_{16 / 17}+2 w_{18}\right)$ \\
& 11 & $\Xi_{c}^{+}, \pi^{0}$ & $g_{2} b_{D / F}\left(w_{16 / 17}+2 w_{18}\right)$ \\
& 12 & $\Xi_{c}^{0}, \pi^{ \pm}$ & $g_{2} b_{D / F} w_{18}$ \\
\hline (g), (h) & 13 & $\Xi_{c}^{\prime+}, \eta_{0}$ & $\beta\left(w_{13 / 14}+2 w_{15}\right)$ \\
\hline
\end{tabular}

Table 9. Loop contribution to the $F_{3}\left(q^{2}\right)$ of the $\Xi_{c}^{\prime+}$ baryon.

\begin{tabular}{|cccc|}
\hline Diagram type & Number & Meson-baryon state & Coefficient \\
\hline (c), (d) & 1 & $\Sigma_{c}^{+}, K^{ \pm}$ & $g_{1} b_{D / F}$ \\
& 2 & $\Xi_{c}^{\prime}, \pi^{ \pm}$ & $g_{1} b_{D / F}$ \\
& 3 & $\Lambda_{c}^{+}, K^{ \pm}$ & $g_{2} b_{D / F}$ \\
& 4 & $\Xi_{c}^{+}, \pi^{ \pm}$ & $g_{2} b_{D / F}$ \\
\hline & 5 & $\Xi_{c}^{\prime 0}, \eta_{8}$ & $g_{1} b_{D / F} w_{18}$ \\
& 6 & $\Xi_{c}^{\prime 0}, \eta_{0}$ & $\beta g_{1} w_{18}$ \\
& 7 & $\Xi_{c}^{\prime 0}, \pi^{0}$ & $g_{1} b_{D / F} w_{18}$ \\
$(\mathrm{e}),(\mathrm{f})$ & 8 & $\Sigma_{c}^{0}, K^{0}$ & $g_{1} b_{D / F} w_{18}$ \\
& 9 & $\Omega_{c}^{0}, K^{0}$ & $g_{1} b_{D / F} w_{18}$ \\
& 10 & $\Lambda_{c}^{0}, K^{ \pm}$ & $g_{2} b_{D / F}\left(w_{16 / 17}+2 w_{18}\right)$ \\
& 11 & $\Xi_{c}^{0}, \eta_{8}$ & $g_{2} b_{D / F} w_{18}$ \\
& 12 & $\Xi_{c}^{0}, \pi^{0}$ & $g_{2} b_{D / F} w_{18}$ \\
\hline (g), (h) & 13 & $\Xi_{c}^{\prime 0}, \eta_{0}$ & $\beta w_{15}$ \\
\hline
\end{tabular}

Table 10. Loop contribution to the $F_{3}\left(q^{2}\right)$ of the $\Xi_{c}^{\prime 0}$ baryon. 


\begin{tabular}{|cccc|}
\hline Diagram type & Number & Meson-baryon state & Coefficient \\
\hline (c), (d) & 1 & $\Xi_{c}^{\prime+}, K^{ \pm}$ & $g_{1} b_{D / F}$ \\
& 2 & $\Xi_{c}^{+}, K^{ \pm}$ & $g_{2} b_{D / F}$ \\
\hline & 3 & $\Omega_{c}^{0}, \eta_{8}$ & $g_{1} b_{D / F} w_{18}$ \\
$(\mathrm{e}),(\mathrm{f})$ & 4 & $\Omega_{c}^{0}, \eta_{0}$ & $\beta g_{1} w_{18}$ \\
& 5 & $\Xi_{c}^{+}, K^{ \pm}$ & $g_{2} b_{D / F}\left(w_{16 / 17}+2 w_{18}\right)$ \\
& 6 & $\Xi_{c}^{0}, K^{0}$ & $g_{2} b_{D / F} w_{18}$ \\
\hline$(\mathrm{g}),(\mathrm{h})$ & 7 & $\Omega_{c}^{0}, \eta_{0}$ & $\beta w_{15}$ \\
\hline
\end{tabular}

Table 11. Loop contribution to the $F_{3}\left(q^{2}\right)$ of the $\Omega_{c}^{0}$ baryon.

\section{Results for the CP-violating form factor}

This appendix contains results for the loop contributions to the form factor $F_{3}\left(q^{2}\right)$ for the various baryons, for photon virtualities below $q^{2} \simeq 0.3 \mathrm{GeV}^{2}$.

\begin{tabular}{|lccccccccc|}
\hline$q^{2}\left(\mathrm{GeV}^{2}\right)$ & $\Lambda_{c}^{+}$ & $\Xi_{c}^{+}$ & $\Xi_{c}^{0}$ & $\Sigma_{c}^{++}$ & $\Sigma_{c}^{+}$ & $\Sigma_{c}^{0}$ & $\Xi_{c}^{\prime+}$ & $\Xi_{c}^{\prime 0}$ & $\Omega_{c}^{0}$ \\
\hline 0.0484 & 1.1028 & 1.1772 & 0.7362 & -0.1150 & 0.1339 & 0.3832 & 0.0361 & 0.2102 & 0.1855 \\
0.1024 & 1.1015 & 1.1764 & 0.7339 & -0.0434 & 0.1359 & 0.3164 & 0.0452 & 0.2047 & 0.1861 \\
0.1444 & 1.1004 & 1.1756 & 0.7323 & -0.0175 & 0.1374 & 0.2943 & 0.0500 & 0.2027 & 0.1865 \\
0.1936 & 1.0992 & 1.1747 & 0.7305 & 0.0021 & 0.1392 & 0.2789 & 0.0546 & 0.2014 & 0.1871 \\
0.2500 & 1.0978 & 1.1735 & 0.7285 & 0.0181 & 0.1412 & 0.2679 & 0.0590 & 0.2007 & 0.1877 \\
0.3136 & 1.0963 & 1.1722 & 0.7264 & 0.0319 & 0.1435 & 0.2598 & 0.0633 & 0.2006 & 0.1885 \\
\hline
\end{tabular}

Table 12. Loop contribution to the $F_{3}\left(q^{2}\right)$ of the $B_{\overline{3}}$ and $B_{6}$ states for $w_{10}^{\prime}, w_{13 / 14}^{\prime}, w_{15}^{\prime}=-0.5$.

\begin{tabular}{|lccccccccc|}
\hline$q^{2}\left(\mathrm{GeV}^{2}\right)$ & $\Lambda_{c}^{+}$ & $\Xi_{c}^{+}$ & $\Xi_{c}^{0}$ & $\Sigma_{c}^{++}$ & $\Sigma_{c}^{+}$ & $\Sigma_{c}^{0}$ & $\Xi_{c}^{\prime+}$ & $\Xi_{c}^{\prime 0}$ & $\Omega_{c}^{0}$ \\
\hline 0.0484 & 0.0245 & 0.0121 & -0.0414 & -0.4594 & -0.1246 & 0.2102 & -0.2403 & 0.0253 & -0.0108 \\
0.1024 & 0.0232 & 0.0113 & -0.0437 & -0.3912 & -0.1252 & 0.1417 & -0.2339 & 0.0181 & -0.0121 \\
0.1444 & 0.0221 & 0.0105 & -0.0453 & -0.3680 & -0.1257 & 0.1183 & -0.2311 & 0.0147 & -0.0130 \\
0.1936 & 0.0209 & 0.0096 & -0.0471 & -0.3513 & -0.1262 & 0.1015 & -0.2289 & 0.0118 & -0.0140 \\
0.2500 & 0.0195 & 0.0085 & -0.0491 & -0.3388 & -0.1268 & 0.0887 & -0.2272 & 0.0094 & -0.0152 \\
0.3136 & 0.0180 & 0.0071 & -0.0512 & -0.3290 & -0.1275 & 0.0786 & -0.2259 & 0.0073 & -0.0165 \\
\hline
\end{tabular}

Table 13. Loop contribution to the $F_{3}\left(q^{2}\right)$ of the $B_{\overline{3}}$ and $B_{6}$ states for $w_{10}^{\prime}, w_{13 / 14}^{\prime}, w_{15}^{\prime}=0$. 


\begin{tabular}{|lccccccccc|}
\hline$q^{2}\left(\mathrm{GeV}^{2}\right)$ & $\Lambda_{c}^{+}$ & $\Xi_{c}^{+}$ & $\Xi_{c}^{0}$ & $\Sigma_{c}^{++}$ & $\Sigma_{c}^{+}$ & $\Sigma_{c}^{0}$ & $\Xi_{c}^{\prime+}$ & $\Xi_{c}^{\prime 0}$ & $\Omega_{c}^{0}$ \\
\hline 0.0484 & -1.0545 & -1.1538 & -0.8155 & -0.8065 & -0.3837 & 0.0391 & -0.5173 & -0.1575 & -0.2053 \\
0.1024 & -1.0558 & -1.1546 & -0.8177 & -0.7416 & -0.3869 & -0.0310 & -0.5135 & -0.1665 & -0.2083 \\
0.1444 & -1.0569 & -1.1553 & -0.8194 & -0.7211 & -0.3893 & -0.0557 & -0.5127 & -0.1712 & -0.2106 \\
0.1936 & -1.0581 & -1.1563 & -0.8212 & -0.7075 & -0.3921 & -0.0741 & -0.5129 & -0.1757 & -0.2133 \\
0.2500 & -1.0595 & -1.1574 & -0.8231 & -0.6986 & -0.3954 & -0.0887 & -0.5139 & -0.1799 & -0.2163 \\
0.3136 & -1.0610 & -1.1588 & -0.8253 & -0.6927 & -0.3991 & -0.1008 & -0.5156 & -0.1841 & -0.2196 \\
\hline
\end{tabular}

Table 14. Loop contribution to the $F_{3}\left(q^{2}\right)$ of the $B_{\overline{3}}$ and $B_{6}$ states for $w_{10}^{\prime}, w_{13 / 14}^{\prime}, w_{15}^{\prime}=0.5$.

Open Access. This article is distributed under the terms of the Creative Commons Attribution License (CC-BY 4.0), which permits any use, distribution and reproduction in any medium, provided the original author(s) and source are credited.

\section{References}

[1] M.B. Gavela, P. Hernández, J. Orloff and O. Pene, Standard model CP-violation and baryon asymmetry, Mod. Phys. Lett. A 9 (1994) 795 [hep-ph/9312215] [INSPIRE].

[2] P. Huet and E. Sather, Electroweak baryogenesis and standard model CP-violation, Phys. Rev. D 51 (1995) 379 [hep-ph/9404302] [INSPIRE].

[3] W. Dekens et al., Unraveling models of CP-violation through electric dipole moments of light nuclei, JHEP 07 (2014) 069 [arXiv: 1404.6082] [INSPIRE].

[4] LHCb collaboration, Observation of CP-violation in Charm Decays, Phys. Rev. Lett. 122 (2019) 211803 [arXiv:1903.08726] [INSPIRE].

[5] LHCb collaboration, A measurement of the CP asymmetry difference in $\Lambda_{c}^{+} \rightarrow p K^{-} K^{+}$and $p \pi^{-} \pi^{+}$decays, JHEP 03 (2018) 182 [arXiv:1712.07051] [INSPIRE].

[6] Y. Grossman and S. Schacht, U-Spin Sum Rules for CP Asymmetries of Three-Body Charmed Baryon Decays, Phys. Rev. D 99 (2019) 033005 [arXiv:1811.11188] [InSPIRE].

[7] LHCb collaboration, Search for $C P$ violation in $\Xi_{c}^{+} \rightarrow p K^{-} \pi^{+}$decays using model-independent techniques, Eur. Phys. J. C 80 (2020) 986 [arXiv:2006.03145] [InSPIRE].

[8] X.-D. Shi, X.-W. Kang, I. Bigi, W.-P. Wang and H.-P. Peng, Prospects for CP and P violation in $\Lambda_{c}^{+}$decays at Super Tau Charm Facility, Phys. Rev. D 100 (2019) 113002 [arXiv: 1904.12415] [inSPIRE].

[9] S. Aiola et al., Progress towards the first measurement of charm baryon dipole moments, arXiv:2010.11902 [INSPIRE].

[10] V. Baluni, CP Violating Effects in QCD, Phys. Rev. D 19 (1979) 2227 [InSPIRE].

[11] R.J. Crewther, P. Di Vecchia, G. Veneziano and E. Witten, Chiral Estimate of the Electric Dipole Moment of the Neutron in Quantum Chromodynamics, Phys. Lett. B 88 (1979) 123 [Erratum ibid. 91 (1980) 487] [INSPIRE].

[12] V. Bernard, Chiral Perturbation Theory and Baryon Properties, Prog. Part. Nucl. Phys. 60 (2008) 82 [arXiv: 0706. 0312] [INSPIRE].

[13] N. Jiang, X.-L. Chen and S.-L. Zhu, Mass and axial charge of heavy baryons, Phys. Rev. D 90 (2014) 074011 [arXiv:1403.5404] [InSPIRE]. 
[14] N. Jiang, X.-L. Chen and S.-L. Zhu, Electromagnetic decays of the charmed and bottom baryons in chiral perturbation theory, Phys. Rev. D 92 (2015) 054017 [arXiv:1505.02999] [INSPIRE].

[15] R.-X. Shi, Y. Xiao and L.-S. Geng, Magnetic moments of the spin-1/2 singly charmed baryons in covariant baryon chiral perturbation theory, Phys. Rev. D 100 (2019) 054019 [arXiv: 1812.07833] [INSPIRE].

[16] J. de Vries, E. Mereghetti, R.G.E. Timmermans and U. van Kolck, The Effective Chiral Lagrangian From Dimension-Six Parity and Time-Reversal Violation, Annals Phys. 338 (2013) 50 [arXiv: 1212.0990] [INSPIRE].

[17] J. Bsaisou, U.-G. Meißner, A. Nogga and A. Wirzba, P- and T-Violating Lagrangians in Chiral Effective Field Theory and Nuclear Electric Dipole Moments, Annals Phys. 359 (2015) 317 [arXiv: 1412.5471] [inSPIRE].

[18] B. Borasoy, The electric dipole moment of the neutron in chiral perturbation theory, Phys. Rev. D 61 (2000) 114017 [hep-ph/0004011] [InSPIRE].

[19] H. Leutwyler, Bounds on the light quark masses, Phys. Lett. B 374 (1996) 163 [hep-ph/9601234] [INSPIRE].

[20] P. Herrera-Siklody, J.I. Latorre, P. Pascual and J. Taron, Chiral effective Lagrangian in the large $N_{c}$ limit: the nonet case, Nucl. Phys. B 497 (1997) 345 [hep-ph/9610549] [INSPIRE].

[21] K. Ottnad, B. Kubis, U.-G. Meißner and F.-K. Guo, New insights into the neutron electric dipole moment, Phys. Lett. B 687 (2010) 42 [arXiv:0911.3981] [InSPIRE].

[22] T.-M. Yan, H.-Y. Cheng, C.-Y. Cheung, G.-L. Lin, Y.C. Lin and H.-L. Yu, Heavy quark symmetry and chiral dynamics, Phys. Rev. D 46 (1992) 1148 [Erratum ibid. 55 (1997) 5851] [INSPIRE].

[23] B. Borasoy and U.-G. Meißner, Chiral Expansion of Baryon Masses and $\sigma$-Terms, Annals Phys. 254 (1997) 192 [hep-ph/9607432] [INSPIRE].

[24] F.-K. Guo and U.-G. Meißner, Baryon electric dipole moments from strong CP-violation, JHEP 12 (2012) 097 [arXiv: 1210.5887] [InSPIRE].

[25] B. Kubis and U.-G. Meißner, Baryon form-factors in chiral perturbation theory, Eur. Phys. J. C 18 (2001) 747 [hep-ph/0010283] [InSPIRE].

[26] U.-G. Meißner and S. Steininger, Baryon magnetic moments in chiral perturbation theory, Nucl. Phys. B 499 (1997) 349 [hep-ph/9701260] [INSPIRE].

[27] P. Herrera-Siklody, J.I. Latorre, P. Pascual and J. Taron, $\eta-\eta^{\prime}$ mixing from $\mathrm{U}(3)_{L} \otimes \mathrm{U}(3)_{R}$ chiral perturbation theory, Phys. Lett. B 419 (1998) 326 [hep-ph/9710268] [InSPIRE].

[28] J. Dragos, T. Luu, A. Shindler, J. de Vries and A. Yousif, Confirming the Existence of the strong CP Problem in Lattice QCD with the Gradient Flow, arXiv:1902.03254 [INSPIRE].

[29] E. Shintani, T. Blum, T. Izubuchi and A. Soni, Neutron and proton electric dipole moments from $N_{f}=2+1$ domain-wall fermion lattice QCD, Phys. Rev. D 93 (2016) 094503 [arXiv: 1512.00566] [INSPIRE].

[30] F.-K. Guo et al., The electric dipole moment of the neutron from 2+1 flavor lattice QCD, Phys. Rev. Lett. 115 (2015) 062001 [arXiv:1502.02295] [INSPIRE].

[31] F. Abusaif et al., Storage Ring to Search for Electric Dipole Moments of Charged Particles - Feasibility Study, arXiv:1912.07881 [INSPIRE]. 